\title{
Improvement of quality of life and mental health in methadone maintenance therapy
}

\begin{abstract}
Background: Methadone Maintenance Therapy (MMT) is an effective harm reduction program. This study aims to identify the impact of MMT program on clients' quality of life and to explore factors that may be associated with mental health.
\end{abstract}

Methods: The study design was a cross-sectional research. 296 participants were recruited randomly from methadone maintenance treatment (MMT) clinics from Mashhad, Iran, in Feb 2015. Quality of life (QoL) was measured by WHOQOL-BREF, and Depression, Anxiety and Stress Scales (DASS) was used for psychological status assessment.

Results: MMT significantly improved social component of WHOQOL-BREF. There is a significant correlation between education levels, marriage status, employment and having chronic disease with score difference in the psychological and social domain of QoL. The difference in education levels, employment and having chronic disease also reached significant levels in environment domain. The differences were statistically significant in higher duration of MMT and all three subscales of DASS scores. QoL was significantly negatively associated with all three domains of DASS scores.

Conclusion: Our study declared mental health was improved by MMT. There was a decrease in all subscales of DASS and associated with QoL.

Keywords: quality of life, opioid related disorders, methadone, substance abuse treatment centers, methadone maintenance treatment, WHOQOL, depression, anxiety stress scale
Volume 3 Issue 3 - 2017

\author{
Mahdi Talebi,' Abbas Zavar,' Mahdi Ghafari, ${ }^{2}$ \\ Razieh Poorandy' \\ 'Mashhad University of Medical Sciences, Iran \\ ${ }^{2}$ Ferdowsi University, Iran
}

Correspondence: Abbas Zavar,Addiction Research Center (ADRC), Imam Reza Hospital, Mashhad University of Medical Sciences (MUMS), Mashhad, Iran, Tel/Fax +985। I85253।5, Email Abbaszavar@gmail.com

Received: August 14, 2016 | Published: April 25, 2017
Abbreviations: MMT, methadone maintenance therapy; WHOQOL, world health organization quality of life assessment; QoL, quality of life; DASS, depression anxiety stress scale.

\section{Introduction}

Drug abuse has long reached a global scale, hitting a number of nations more severely. ${ }^{1,2}$ Opioid use in Iran has been commonplace ${ }^{3,4}$ both for cultural and historical reasons; ${ }^{5}$ and perhaps more importantly, adjacency with the world largest opium producer: Afghanistan. ${ }^{6,7}$ Two million Iranians use $450 \mathrm{mt}$ of opioids annually, almost $42 \%$ of the entire global consumption. ${ }^{6-8}$ Owing to the high morbidity and mortality addicts are often infected with, which are known to be ongoing during years of dependence, addition can be considered as a chronic condition. ${ }^{9,10}$

Proposed by WHO as the efficient therapeutic modality in opioid dependence, MMT (Methadone Maintenance Therapy) has been implemented as an effective harm reduction programme the world over, ${ }^{11,12}$ practiced in Iran since 2003. Korasan Razavi, a northern state with joined borders with Afghanistan, accommodates more than 500 MMT clinics.

By definition, quality of life is regarded as "An individual's perception of their position in life in the context of the culture and value systems in which they live and in relation to their goals, expectations, standards and concerns", ${ }^{13}$ the assessment and/or quantification of which has recently drawn particular attention in the realm of healthcare, particularly as an index for drug programmers evolution. ${ }^{1,14-16}$
As our initial target, we strived to measure addicts' quality of life following their MMT programme implemented on an out patients basis. We also intended to explore the mental parameters associated with the changes in their quality of life.

\section{Materials and methods}

\section{Setting}

In this cross-section study, we recruited subjects receiving methadone from MMT clinics across the state capital city of Mashhad in Khorasan Razavi province on an outpatient basis during the month of February, 2015.

\section{Participants}

Thirty MMT clients were selected at random from each of the 505 MMT clinics using a random number table, yielding a total sample size of 300. 296 participants were recruited randomly from MMT clinics who met eligibility criteria were enrolled into the study. The refusal rate was less than $3 \%$.

\section{Inclusion and exclusion criteria}

We included those with an ICD-10 diagnosis of current opioid dependence who were between is to 70 years of age. Prior to signing the informed consent, their mental competence had been evaluated by a clinician. They were also physically capable of participating in our assessment programme in terms of their overall health.

We had to exclude those with a mental retardation or cognitive impairment. Any subject with severe psychotic or behavioral 
disturbance was also ruled out. Some failed as they did not manage to fill in the questionnaire for a variety of reasons while those who had received other models of treatment for their drug use were not registered either.

Those on the list, a total of 296 out of 300 , were given a selfadministered questionnaire to complete following an interview with staff regarding their question as the confidentiality of the information given and other possible dork areas in this respect. They all signed an informed consent form as well.

\section{Data collection tools and measurements}

Socio-demographic: Social demographic data such as age at the time of enrolment, gender, marital status, employment and formal education were noted. Other variables of interest were health status, the age of drug commencement, duration and the kind of illicit drug use.

Quality of life: This assessment utilizes the WHO Quality of Life questionnaire (WHOQOL). ${ }^{17}$ The WHOQOL-BREF is a shorter version of the original instrument that may be more convenient for use in large research studies or clinical trials. ${ }^{13,18}$ The WHOQOLBREF instrument consists of 26 items, measuring the following broad domains: Physical health activities of daily living, Psychological bodily image and appearance, Social and personal relationships, and Environmental-financial resources.

The administration of the World Health Organization Quality of Life-BREF (WHOQOL-BREF) to MMT clients was assisted by trained health personnel to ensure that clients fully understand the questionnaire. A high score represents good quality of life. An increase in QoL scores after 3, 6 or 12 months of treatment would suggest the effectiveness of MMT.

Domain scores for the WHOQOL-BREF are calculated by taking the means of all items included in each domain, multiplied by a factor of four. The original domain scores were transformed to a scale of $0-100$ according to the equation in the published guidelines. ${ }^{17}$ This questionnaire is appropriate for the assessment of outpatients receiving MMT.9,16,19

The validity, reliability, internal consistency, and dimensional structure of the Farsi version of WHOQOL-BREF in an Iranian population was approved (Cronbach's alpha $=0.70){ }^{20,21}$

\section{The short form of depression anxiety stress scales (DASS-2I)}

The Depression, Anxiety and Stress Scales (DASS) ${ }^{22}$ is a widely used screening tool and comprises three sub-scales in community settings:

a. The Depression sub-scale which measures hopelessness, low self-esteem, and low positive affect;

b. The Anxiety scale which assesses autonomic arousal, muscular-skeletal symptoms, situational anxiety and subjective experience of anxious arousal;

c. The Stress scale which assesses tension, agitation, and negative affect.

There are two forms of the DASS, the full 42-item and the short 21 -item versions, both of which assess the same domains. The short version has, ${ }^{21}$ which has been shown to have high internal consistency. The validated Farsi version of DASS- 21 was used in this study. ${ }^{15,23-25}$ Respondents were asked to rate their experience on each symptom over the past week on a 4-point severity scale ranging from 0 (does not apply to me), to 3 (applies to me most or all of the time). Scores pertaining to each scale were later summed up and categorized as normal, mild, moderate, severe and extremely severe according to the DASS Manual..$^{22}$ The combination of these two questionnaires was applied in some studies. ${ }^{15,24,26}$

\section{Statistical analysis}

The scoring from the WHOQOL-BREF (raw score) was transformed to the $0-100$ scale scoring format, following to the guidelines for the transformation of raw WHOQOL-BREF score, which is available from the WHOQOL Group. ${ }^{17}$ The end result provided scores in the transformed 0-100 scale format for each of the four quality of life domains: physical, psychological, social relationships and environment.

Statistical analyses were performed using SPSS-11.5 for both descriptive and inferential analysis. Univariate statistics such as mean values, standard deviations, frequencies and proportion percentages were derived for continuous and categorical variables respectively. Bivariate and multivariate analyses were used to measure the association strength between the variables in the study and identify predictors for the outcomes of interest respectively. Multiple regression models were applied to the four domains of quality-oflife score and DASS score, controlling for the simultaneous effect of demographic characteristics. Standardized regression coefficients and their significance levels are reported. The level of statistical significance was set at $\mathrm{p}<0.05$.

\section{Results}

The background characteristics of the participants are summarized in Table 1. As can be seen, the age ranged from 17 to 71 years old with mean of $37.8 \pm 10.1 .81 .4 \%$ of participates were male. More than a third whereas a mere $3 \%$ were registered as illiterate. Unemployment rate, however, reached a staggering $68 \%$. The mean age (SD; MinMax) at the onset of regular drug use was $29.5(8.1 ; 8-53)$ years while the mean duration of substances dependency was $8.3(4.9 ; 1-35)$ years.

Participant had been undergoing MMT for an average of $14.3 \pm 10.3$ months, ranging from 1 week to 57 months. The mean methadone dose for the substitution treatment throughout the therapy was $55 \pm 15 \mathrm{mg}$.

Table 2 shows the changes of QoL score and subscale scores of outpatients receiving MMT as treatment commenced and after 3, 6 and 12 months. The methadone maintenance treatment significantly improved the social component of WHOQOL-BREF ( $\mathrm{P}=0.008)$ whereas there could be found any considerable change in the two .i.e. psychological and environmental. As can be seen in Figure 1, a minor increase is apparent comparing baseline values and those of month 12 , which signifies quality of life enhancement throughout and after methadone therapy.

Univariate analysis was done to identify variables that are associated with changes in each domain of the quality of life. Significant results for each domain are shown in Table 3. There can be seen a significant correlation between education levels, marriage status, employment and having a chronic disease, with score difference in the psychological and social domains. The pertinent values were 
also considerable in the environment domain. Based on ANOVA bivariate analysis outcome, patients also differed in their education $(\mathrm{p}=0.00)$, substance kind $(\mathrm{p}=0.01)$ and marital status $(\mathrm{p}=0.02)$ when assessed for quality of life. Other influential parameters, based on $t$-test calculation, included employment $(\mathrm{P}=0.00)$ and an underling chronic condition $(\mathrm{P}=0.01)$.

Table I Descriptive characteristics of the study participants $(n=296)$

\begin{tabular}{lll}
\hline Variable & Frequency & $\%$ \\
\hline Gender & & \\
\hline Male & 241 & 81.4 \\
Female & 55 & 18.6 \\
\hline Age (years) & & \\
\hline$<20$ & 4 & 1.4 \\
$20-30$ & 73 & 24.7 \\
$30-40$ & 117 & 39.5 \\
$40-50$ & 68 & 23 \\
$>50$ & 34 & 11.5 \\
\hline Education status & & \\
\hline Illiterate & 8 & 2.7 \\
Under-Diploma & 110 & 37.2 \\
Diploma & 116 & 39.2 \\
Academic & 62 & 20.9 \\
\hline Marriage status & & \\
\hline Single & 52 & 17.6 \\
Married & 231 & 78 \\
Divorced/Widow & 13 & 4.4 \\
\hline Occupational status & & \\
\hline Employed & 228 & 77 \\
Unemployed & 68 & 23 \\
\hline Kind of substance use & \\
\hline Opium & 172 & 58.1 \\
Heroin & 69 & 23.3 \\
Tramadol & 14 & 4.7 \\
Poly Drug User & 41 & 13.9 \\
\hline Wile DASS sco & & \\
\hline
\end{tabular}

This is while DASS scores implied that depression, anxiety and stress were strongly linked with marriage and occupational status as well as a chronic disease (Table 4). This association was also

Table $2 \mathrm{WHOQOL}$ and DASS scores by domain at baseline, 3,6 and 12 months after MMT

\begin{tabular}{|c|c|c|c|c|c|c|c|}
\hline \multicolumn{2}{|c|}{ Questionnaire } & \multirow{2}{*}{$\begin{array}{l}\begin{array}{l}\text { Baseline } \\
\text { (mean } \pm S D)\end{array} \\
20.39 \pm 0.65\end{array}$} & \multirow{2}{*}{$\begin{array}{l}\text { 3-month } \\
\text { MMT } \\
20.43 \pm 0.64\end{array}$} & \multirow{2}{*}{$\begin{array}{l}\text { 6-month } \\
\text { MMT } \\
20.73 \pm 0.5\end{array}$} & \multirow{2}{*}{$\begin{array}{l}\text { I2-month } \\
\text { MMT } \\
21.07 \pm 0.29\end{array}$} & \multirow{2}{*}{$\begin{array}{l}\begin{array}{l}\text { Stat. } \\
\text { method }\end{array} \\
K-W^{\infty}\end{array}$} & \multirow{2}{*}{$\begin{array}{l}P \\
\text { value } \\
0.833\end{array}$} \\
\hline WHOQOL & Physical Health & & & & & & \\
\hline & Psychological Health & $18.95 \pm 0.65$ & $19.68 \pm 0.55$ & $19.65 \pm 0.49$ & $19.75 \pm 0.26$ & $\mathrm{~K}-\mathrm{W}$ & 0.856 \\
\hline & Social Health & $8.98 \pm 0396$ & $8.8 \pm 0.377$ & $9.88 \pm 0.365$ & $9.95 \pm 0.2$ & $\mathrm{~K}-\mathrm{W}$ & $0.008 *$ \\
\hline & Environmental Health & $25.37 \pm 0.95$ & $25.18 \pm 0.89$ & $25.82 \pm 0.82$ & $25.77 \pm 0.43$ & ANOVA & 0.915 \\
\hline & QoL (Total) & $73.68 \pm 2.35$ & $74.09 \pm 1.95$ & $76.11 \pm 1.95$ & $76.55 \pm 0.99$ & ANOVA & 0.527 \\
\hline \multirow[t]{3}{*}{ DASS } & Depression & $27.7 I \pm I .55$ & $28.59 \pm 1.37$ & $24.9 \pm 1.1$ & $24.85 \pm 0.7$ & $\mathrm{~K}-\mathrm{W}$ & $0.003 *$ \\
\hline & Anxiety & $26.73 \pm 1.17$ & $26.23 \pm 1.3$ & $23.77 \pm 1.06$ & $22.75 \pm 0.64$ & $\mathrm{~K}-\mathrm{W}$ & $0.003^{*}$ \\
\hline & Stress & $30.39 \pm 1.55$ & $31.73 \pm 1.39$ & $28.27 \pm 1.2$ & $26.5 I \pm 0.67$ & $\mathrm{~K}-\mathrm{W}$ & $0.036 *$ \\
\hline
\end{tabular}

*Significant when $\mathrm{P}$ value $<0.05$

ФK-W: Kruksal-Wallis. significant only between education levels and anxiety and stress subscale. As was illustrated in Table 2 and Figure 2, the differences were statistically significant in longer durations of MMT and all three subscales of DASS scores $(\mathrm{p}=0.003, \mathrm{p}=0.003$ and $\mathrm{p}=0.036$ for depression, anxiety and stress subscales respectively).

Table 5 presents the correlation between coefficients of variables. Addiction duration was negatively associated with of being single education $(\mathrm{r}=-0.17)$ and positively associated with age $(\mathrm{r}=0.5)$ and state married $(\mathrm{r}=0.16)$. Unemployment status was associated with lower physical $(\mathrm{r}=-0.15)$, psychological $(\mathrm{r}=-0.20)$, social $(\mathrm{r}=-0.14)$, environmental $(\mathrm{r}=-0.17)$ health and QoL $(\mathrm{r}=-0.19)$. Participants with higher education levels reported a significantly higher psychological $(r=-0.20)$, social $(r=-0.20)$ and environmental $(r=-0.32)$ health and finally with QoL ( $\mathrm{r}=-0.25)$. The quality of life was affected by a having chronic disease $(\mathrm{r}=0.10)$. It was also inversely associated with all three domains of DASS scores $(r=-0.50$ for the stress subscale, $\mathrm{r}=-0.44$ for the anxiety subscale and $\mathrm{r}=-0.50$ for the depression subscale).

Unemployment status was directly associated with three domains of DASS scores $(\mathrm{r}=0.18, \mathrm{r}=0.19$ and $\mathrm{r}=0.20$ for stress, anxiety and depression subscales in order).

The results of multiple regression analyses are presented in Table 6. The first four columns of the table summarize findings from the multiple linear regression models in four domains of quality of life, controlling for background characteristics.

Unemployment was negatively associated with all four domains of quality of life $(\beta=-0.155, \beta=-0.168, \beta=-0.121$ and $\beta=-0.144$ for physical, psychological, social and environmental health, respectively). Education was also proved to have directly affected psychological $(\beta=0.204)$, social $(\beta=0.302)$ and environmental health $(\beta=0.302)$.

Table 6 displays that increasing of MMT duration was negatively associated with domains of stress $(\beta=-0.145)$ and anxiety subscale $(\beta=-0.147)$. Likewise, DASS subscale educational, anxiety $(\beta=-0.141)$ and depression $(\beta=-0.219)$ were adversely influence by a chronic condition. Nevertheless, a positive correlation was established between unemployment and the above three (stress $\beta=0.174$, anxiety $\beta=0.143$ and $\beta=0.159$ ).

WK-Wruksal-Wallis.

Citation: Talebi M, Zavar A, Ghafari M, et al. Improvement of quality of life and mental health in methadone maintenance therapy. MOJ Addict Med Ther. 20I7;3(3):82-89. DOI: 10.15406/mojamt.2017.03.00039 
Table 3 Univariate analysis showing significant variables associated with improvement in each quality of life domain

\begin{tabular}{|c|c|c|c|c|c|c|c|c|c|c|}
\hline \multirow{3}{*}{$\begin{array}{l}\text { Questionnaire } \\
\text { Variable }\end{array}$} & \multicolumn{10}{|c|}{ WHOQOL } \\
\hline & \multicolumn{2}{|c|}{ Physical health } & \multicolumn{2}{|c|}{$\begin{array}{l}\text { Psychological } \\
\text { health }\end{array}$} & \multicolumn{2}{|c|}{ Social health } & \multicolumn{2}{|c|}{$\begin{array}{l}\text { Environmental } \\
\text { health }\end{array}$} & \\
\hline & Meant & $\mathrm{P}$ & Mean $\pm S D$ & $\mathrm{P}$ & Mean $\pm S D$ & $\mathrm{P}$ & mean $\pm S D$ & $\mathrm{P}$ & mean $\pm S D$ & $P$ \\
\hline Gender: & M-W & 0.44 & $\mathbf{M}-\mathbf{W}$ & 0.76 & M-W & 0.95 & T-test & 0.69 & T-test & 0.95 \\
\hline Male & \multicolumn{2}{|c|}{$20.7 I \pm 0.25$} & \multicolumn{2}{|l|}{$19.66 \pm 0.23$} & \multicolumn{2}{|l|}{$9.64 \pm 0.16$} & \multicolumn{2}{|l|}{$25.7 \pm 0.35$} & \multicolumn{2}{|l|}{$75.7 I \pm 0.85$} \\
\hline Female & \multicolumn{2}{|c|}{$21.24 \pm 0.48$} & \multicolumn{2}{|l|}{$|9.4| \pm 0.45$} & \multicolumn{2}{|l|}{$9.57 \pm 0.34$} & \multicolumn{2}{|l|}{$25.36 \pm 0.85$} & \multicolumn{2}{|l|}{$75.59 \pm 1.86$} \\
\hline Age (years) & $\mathbf{K}-\mathbf{W v}$ & 0.95 & $\mathbf{K}-\mathbf{W}$ & 0.23 & K-W & 0.56 & ANOVA & 0.65 & ANOVA & 0.66 \\
\hline$<20$ & \multicolumn{2}{|c|}{$20.00 \pm 0.00$} & \multicolumn{2}{|l|}{$18.00 \pm 0.00$} & \multicolumn{2}{|l|}{$9.00 \pm 0.00$} & \multicolumn{2}{|l|}{$24.00 \pm 0.00$} & \multicolumn{2}{|l|}{$71.00 \pm 0.00$} \\
\hline $30-40$ & $20.94 \pm$ & & $20.06 \pm 0.30$ & & $9.81 \pm 0.23$ & & $26.22 \pm 0.48$ & & $77.00 \pm 1.14$ & \\
\hline $40-50$ & $20.76 \pm$ & & $\mid 9.21 \pm 0.42$ & & $9.42 \pm 0.28$ & & $24.96 \pm 0.58$ & & $74.32 \pm 1.49$ & \\
\hline$>50$ & $20.67 \pm$ & & $19.04 \pm 0.55$ & & $9.42 \pm 0.43$ & & $25.4 I \pm 1.03$ & & $74.5 I \pm 2.36$ & \\
\hline Education Status & K-W & 0.08 & K-W & $0.00 *$ & K-W & $0.002 *$ & ANOVA & $0.00 *$ & ANOVA & $0.00 *$ \\
\hline Illiterate & $18.13 \pm$ & & $16.51 \pm 1.75$ & & $7.25 \pm 1.61$ & & $19.13 \pm 3.59$ & & $61.00 \pm 8.17$ & \\
\hline Under-Diploma & $20.87 \pm$ & & $19.25 \pm 0.36$ & & $9.29 \pm 0.24$ & & $24.2 I \pm 0.53$ & & $73.61 \pm 1.32$ & \\
\hline Diploma & $20.62 \pm$ & & $|9.32 \pm 0.3|$ & & $9.59 \pm 0.21$ & & $25.94 \pm 0.45$ & & $75.47 \pm 1.11$ & \\
\hline Academic & $21.41 \pm$ & & $21.23 \pm 0.33$ & & $|0.6| \pm 0.3 \mid$ & & $28.47 \pm 0.66$ & & $81.77 \pm 1.34$ & \\
\hline Marriage status & K-W & 0.12 & K-W & $0.009 *$ & K-W & $0.045 *$ & ANOVA & 0.11 & ANOVA & $0.02 *$ \\
\hline Married & $21.05 \pm$ & & $|9.87 \pm 0.2|$ & & $9.74 \pm 0.16$ & & $25.17 \pm 0.36$ & & $76.37 \pm 0.84$ & \\
\hline Divorced/Widow & $19.69 \pm$ & & $\mid 6.62 \pm 1.01$ & & $7.54 \pm 0.83$ & & $22.46 \pm 2.05$ & & $66.31 \pm 4.58$ & \\
\hline $\begin{array}{l}\text { Occupational } \\
\text { status }\end{array}$ & M-W & 0.09 & M-W & $0.01 *$ & M-W & $0.01 *$ & T-test & $0.001 *$ & T-test & $0.00 *$ \\
\hline Employed & $21.12 \pm$ & & $20.03 \pm 0.21$ & & $9.87 \pm 0.16$ & & $26.21 \pm 0.34$ & & $77.23 \pm 0.81$ & \\
\hline Unemployed & $19.74 \pm$ & & $18.25 \pm 0.47$ & & $8.82 \pm 0.35$ & & $23.72 \pm 0.83$ & & $70.53 \pm 1.93$ & \\
\hline $\begin{array}{l}\text { Kind of substance } \\
\text { use }\end{array}$ & K-W & 0.79 & K-W & 0.01 & K-W & 0.22 & ANOVA & $0.03 *$ & ANOVA & $0.01 *$ \\
\hline Opium & $20.81 \pm$ & & $19.94 \pm 0.25$ & & $9.73 \pm 0.19$ & & $25.6 I \pm 0.4 I$ & & $76.09 \pm 0.96$ & \\
\hline Heroin & $20.77 \pm$ & & $19.13 \pm 0.43$ & & $9.71 \pm 0.31$ & & $25.33 \pm 0.68$ & & $74.94 \pm 1.58$ & \\
\hline Methamphetamine & $20.6 \pm 1$ & & $19.61 \pm 2.23$ & & $8.21 \pm 1.02$ & & $22.00 \pm 3.24$ & & $70.4 I \pm 7.22$ & \\
\hline Tramadol & $22.93 \pm$ & & $22.00 \pm 0.62$ & & $10.79 \pm 0.72$ & & $29.86 \pm 1.24$ & & $85.57 \pm 2.63$ & \\
\hline Poly Drug User & $19.83 \pm$ & & $18.03 \pm 0.67$ & & $8.53 \pm 0.47$ & & $25.00 \pm 1.10$ & & $95.00 \pm 0.00$ & \\
\hline Chronic disease & M-W & 0.15 & M-W & $0.04^{*}$ & M-W & $0.04 *$ & T-test & $0.009 *$ & T-test & $0.01 *$ \\
\hline Yes & $19.81 \pm$ & & $18.19 \pm 0.89$ & & $8.62 \pm 0.48$ & & $22.52 \pm 1.31$ & & $69.14 \pm 3.09$ & \\
\hline No & $20.89 \pm$ & & $|9.72 \pm 0.2|$ & & $9.71 \pm 0.17$ & & $25.88 \pm 0.33$ & & $76.19 \pm 0.79$ & \\
\hline
\end{tabular}

*Significant when $\mathrm{P}$ value $<0.05$

œ-W: Mann-Whitney

ФK-W: Kruksal-Wallis

Citation: Talebi M, Zavar A, Ghafari M, et al. Improvement of quality of life and mental health in methadone maintenance therapy. MOJ Addict Med Ther. 2017;3(3):82-89. DOI: 10.15406/mojamt.2017.03.00039 
Table 4 Univariate analysis showing significant variables associated with improvement in each DASS domain

\begin{tabular}{|c|c|c|c|c|c|c|}
\hline \multirow{3}{*}{$\begin{array}{l}\text { Questionnaire } \\
\text { Variable }\end{array}$} & \multicolumn{6}{|l|}{ DASS } \\
\hline & \multicolumn{2}{|c|}{ Depression } & \multicolumn{2}{|l|}{ Anxiety } & \multicolumn{2}{|l|}{ Stress } \\
\hline & mean $\pm S D$ & $\mathrm{P}$ & mean $\pm S D$ & $\mathrm{P}$ & mean $\pm S D$ & $\mathrm{P}$ \\
\hline Gender: & M-WX & 0.11 & M-W & 0.06 & M-W & 0.57 \\
\hline Male & $27.73 \pm 0.56$ & & $23.42 \pm 0.48$ & & $25.58 \pm 0.55$ & \\
\hline Female & $30.19 \pm 1.31$ & & $26.74 \pm|.4|$ & & $26.89 \pm 1.36$ & \\
\hline Age (years) & K-Wv & 0.74 & K-W & 0.66 & K-W & 0.95 \\
\hline$<20$ & $26.00 \pm 0.00$ & & $26.00 \pm 0.00$ & & $28.00 \pm 0.00$ & \\
\hline $20-30$ & $29.38 \pm 1.28$ & & $24.27 \pm 0.69$ & & $26.44 \pm 1.23$ & \\
\hline $30-40$ & $28.33 \pm 0.75$ & & $23.5 I \pm 0.97$ & & $25.59 \pm 0.75$ & \\
\hline $40-50$ & $27.5 I \pm 1.13$ & & $25.24 \pm|.4|$ & & $25.47 \pm 1.07$ & \\
\hline$>50$ & $27.33 \pm 1.37$ & & $25.24 \pm|.4|$ & & $26.00 \pm 1.51$ & \\
\hline Education status & K-W & 0.14 & K-W & $0.02 *$ & K-W & $0.00 I^{*}$ \\
\hline Illiterate & $32.25 \pm 3.82$ & & $33.75 \pm 4.82$ & & $37.00 \pm 4.99$ & \\
\hline Under-Diploma & $28.55 \pm 0.89$ & & $24.84 \pm 0.78$ & & $27.07 \pm 0.86$ & \\
\hline Diploma & $28.19 \pm 0.81$ & & $23.59 \pm 0.70$ & & $25.52 \pm 0.79$ & \\
\hline Academic & $26.58 \pm 1.01$ & & $22.12 \pm 0.92$ & & $23.58 \pm 0.87$ & \\
\hline Marriage status & K-W & $0.003 *$ & K-W & $0.007 *$ & K-W & 0.008* \\
\hline Single & $29.73 \pm 1.27$ & & $24.08 \pm 1.12$ & & $27.84 \pm 1.32$ & \\
\hline Married & $27.48 \pm 0.58$ & & $23.58 \pm 0.52$ & & $24.95 \pm 0.55$ & \\
\hline Divorced/Widow & $34.62 \pm 1.79$ & & $31.85 \pm 2.76$ & & $33.23 \pm 3.29$ & \\
\hline Occupational status & M-W & $0.001 *$ & M-W & $0.00 I^{*}$ & M-W & 0.00 I* \\
\hline Employed & $24.74 \pm 0.54$ & & $22.98 \pm 0.47$ & & $27.11 \pm 0.53$ & \\
\hline Unemployed & $29.32 \pm 1.25$ & & $27.47 \pm 1.22$ & & $31.74 \pm 1.29$ & \\
\hline Kind of substance use & K-W & 0.29 & K-W & 0.05 & K-W & 0.11 \\
\hline Opium & $27.2 I \pm 0.67$ & & $23.4 I \pm 0.64$ & & $24.98 \pm 0.66$ & \\
\hline Heroin & $28.87 \pm 1.09$ & & $24.43 \pm 0.89$ & & $26.96 \pm 1.09$ & \\
\hline Methamphetamine & $32.80 \pm 5.35$ & & $28.4 I \pm 4.26$ & & $30.8 I \pm 4.92$ & \\
\hline Tramadol & $25.7 I \pm 2.34$ & & $21.86 \pm 2.07$ & & $20.7| \pm| .8 \mid$ & \\
\hline Poly Drug User & $32.29 \pm 1.43$ & & $26.63 \pm 1.31$ & & $29.4 I \pm 1.46$ & \\
\hline Chronic disease & M-W & $0.00 *$ & M-W & $0.00 *$ & M-W & $0.00 *$ \\
\hline Yes & $32.29 \pm 1.91$ & & $30.76 \pm 1.95$ & & $34.86 \pm 1.97$ & \\
\hline No & $25.29 \pm 0.52$ & & $23.51 \pm 0.48$ & & $27.67 \pm 0.52$ & \\
\hline
\end{tabular}

*Significant when $\mathrm{P}$ value $<0.05$

ФM-W: Mann-Whitney

ळK-W: Kruksal-Wallis

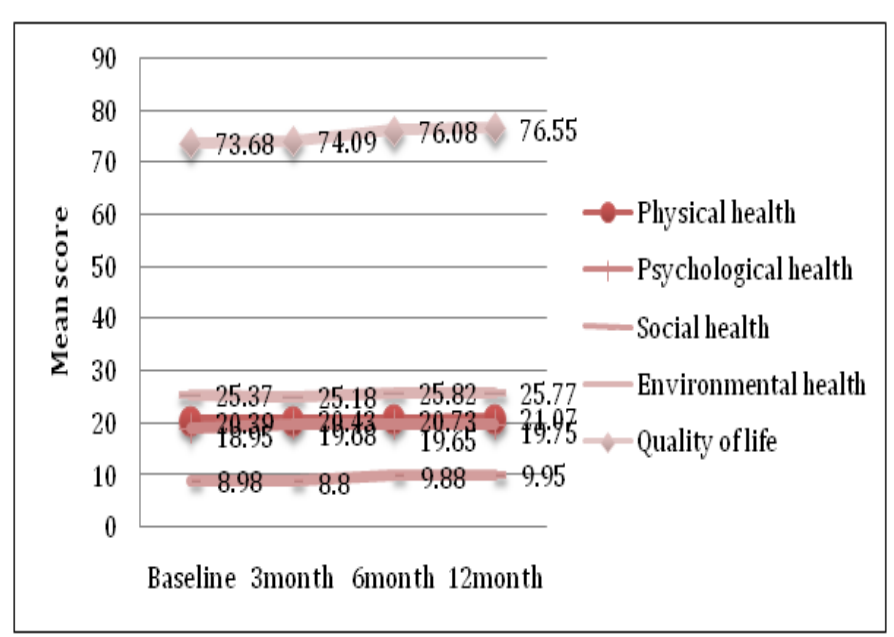

Figure I Quality of life subscale scores changes.

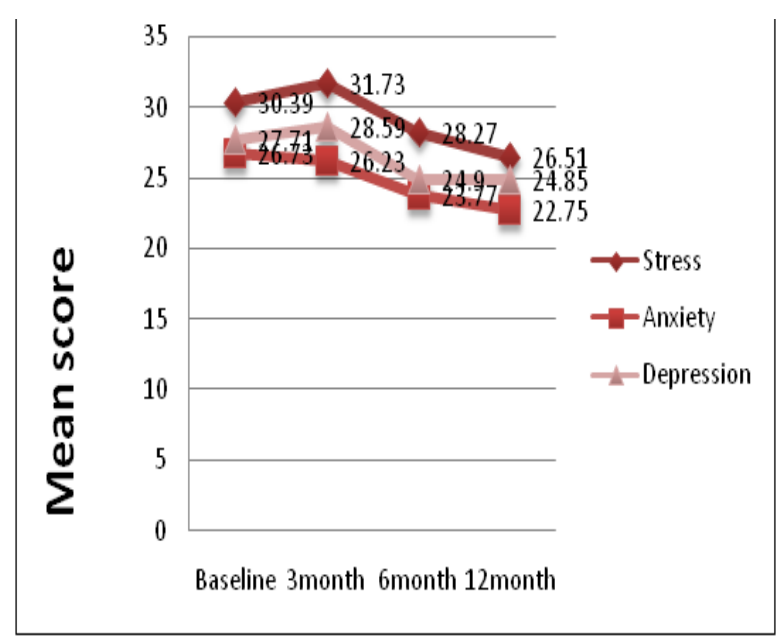

Figure 2 Depression Anxiety Stress Scale changes.

Citation: Talebi M, Zavar A, Ghafari M, et al. Improvement of quality of life and mental health in methadone maintenance therapy. MOJ Addict Med Ther. 2017;3(3):82-89. DOI: I0.15406/mojamt.2017.03.00039 
Table 5 Correlation coefficients and level of significance for background characteristics, quality of life domains and DASS subscales

\begin{tabular}{|c|c|c|c|c|c|c|c|c|c|c|c|c|c|c|c|}
\hline & I & 2 & 3 & 4 & 5 & 6 & 7 & 8 & 9 & 10 & II & 12 & 13 & 14 & 15 \\
\hline \multicolumn{16}{|l|}{ Male } \\
\hline Age (years) & 0.003 & & & & & & & & & & & & & & \\
\hline $\begin{array}{l}\text { Education } \\
\text { Levels }\end{array}$ & -0.05 & $-0.2 * *$ & & & & & & & & & & & & & \\
\hline Single & $0.14^{*}$ & $0.34^{* *}$ & -0.04 & & & & & & & & & & & & \\
\hline Employd & $0.19^{* *}$ & $0.1 *$ & $0.15^{*}$ & 0.03 & & & & & & & & & & & \\
\hline Having & & & & & & & & & & & & & & & \\
\hline $\begin{array}{l}\text { Chronic } \\
\text { Disease }\end{array}$ & -0.04 & $0.2 * *$ & $-0.15^{* *}$ & 0.06 & $0.16 * *$ & & & & & & & & & & \\
\hline $\begin{array}{l}\text { Substance } \\
\text { Kind }\end{array}$ & -0.11 & $-0.3 * *$ & 0.09 & $-0.25^{* *}$ & 0.03 & -0.02 & & & & & & & & & \\
\hline $\begin{array}{l}\text { Addiction } \\
\text { Duration }\end{array}$ & 0.04 & $0.5^{* *}$ & $-0.17 * *$ & $0.16 * *$ & 0.07 & -0.03 & $-0.19 * *$ & & & & & & & & \\
\hline $\begin{array}{l}\text { Physical } \\
\text { Health }\end{array}$ & 0.05 & -0.03 & 0.09 & 0.06 & $-0.15^{*}$ & 0.08 & 0.001 & -0.06 & & & & & & & \\
\hline $\begin{array}{l}\text { Psychology } \\
\text { Health }\end{array}$ & -0.02 & -0.08 & $0.2 * *$ & -0.03 & $-0.2^{* *}$ & $0.12 *$ & -0.09 & -0.09 & $0.6^{* *}$ & & & & & & \\
\hline Social Health & -0.004 & -0.07 & $0.2^{* *}$ & -0.07 & $-0.14 *$ & $0.1^{*}$ & -0.05 & -0.05 & $0.55^{* *}$ & $0.61 * *$ & & & & & \\
\hline $\begin{array}{l}\text { Environmental } \\
\text { Health }\end{array}$ & -0.02 & -0.05 & $0.32 * *$ & -0.07 & $-0.17^{* *}$ & $0.14 *$ & 0.02 & -0.04 & $0.63^{* *}$ & $0.63^{* *}$ & $0.6 * *$ & & & & \\
\hline QoL (total) & -0.003 & -0.06 & $0.25 * *$ & -0.04 & $-0.19 * *$ & $0.1^{*}$ & -0.03 & -0.06 & $0.83 * *$ & $0.83 * *$ & $0.89 * *$ & $0.89 * *$ & & & \\
\hline Stress & 0.09 & -0.1 & -0.09 & -0.001 & $0.18^{* *}$ & $-0.2^{* * *}$ & $0.1^{* *}$ & -0.02 & $-0.35^{* *}$ & $-0.35^{* *}$ & $-0.48 * *$ & $-0.43 * *$ & $-0.5^{* *}$ & & \\
\hline Anxiety & 0.11 & 0.03 & $-0.16 * *$ & 0.07 & $0.19 * *$ & $-0.2^{* *}$ & $0.13^{*}$ & -0.001 & $-0.3 * *$ & $-0.35 * *$ & $-0.44 * *$ & $-0.42 * *$ & $-0.44 * *$ & $0.28 * *$ & \\
\hline Depression & 0.03 & -0.03 & $-0.21 * *$ & -0.03 & $0.2 * *$ & $-0.2^{* *}$ & $0.12 *$ & 0.03 & $-0.34^{* *}$ & $-0.46 * *$ & $-0.53 * *$ & $-0.49 * *$ & $-0.5^{* *}$ & $0.8^{* *}$ & $0.75^{* * *}$ \\
\hline
\end{tabular}

Table 6 Multiple regressions on quality of life and DASS by domain

\begin{tabular}{|c|c|c|c|c|c|c|c|c|}
\hline \multirow[t]{2}{*}{ Questionnaire } & \multicolumn{5}{|c|}{ WHOQOL } & \multicolumn{3}{|l|}{ DASS } \\
\hline & $\begin{array}{l}\text { Physical } \\
\text { health }\end{array}$ & $\begin{array}{l}\text { Psychology } \\
\text { health }\end{array}$ & $\begin{array}{l}\text { Social } \\
\text { health }\end{array}$ & $\begin{array}{l}\text { Environmental } \\
\text { health }\end{array}$ & $\begin{array}{l}\text { QoL } \\
\text { (Total) }\end{array}$ & Stress & Anxiety & Depression \\
\hline Variable & $\beta$ & $\beta$ & $\beta$ & $\beta$ & $\beta$ & $\beta$ & $\beta$ & $\beta$ \\
\hline $\begin{array}{l}\text { Duration of } \\
\text { MMT }\end{array}$ & 0.052 & 0.025 & $0.134 *$ & 0.007 & 0.053 & $0.145^{*}$ & $0.147^{* *}$ & -0.102 \\
\hline Gender & 0.077 & 0.017 & 0.003 & 0.043 & 0.057 & 0.066 & \multirow{2}{*}{$\begin{array}{l}0.114^{*} \\
-0.012\end{array}$} & 0.001 \\
\hline Age & 0.019 & 0.008 & 0.014 & 0.011 & 0.062 & \multirow{2}{*}{$\begin{array}{l}0.156^{*} \\
-0.101\end{array}$} & & -0.073 \\
\hline Education & 0.057 & $0.204 * *$ & $0.204^{* *}$ & $0.302 * *$ & $0.242 * *$ & & $0.14 I^{*}$ & $0.219 * *$ \\
\hline Marriage & 0.045 & -0.153 & -0.098 & -0.011 & -0.072 & 0.063 & \multirow{2}{*}{$\begin{array}{l}0.099 \\
0.143^{*}\end{array}$} & 0.026 \\
\hline Occupation & $0.155^{*}$ & $0.168 * *$ & $0.121 *$ & $0.144 *$ & $0.177 * *$ & $0.174 * *$ & & $0.159 * *$ \\
\hline Substance kind & 0.016 & 0.102 & 0.08 & 0.032 & -0.018 & 0.083 & 0.091 & 0.058 \\
\hline Chronic Dis & 0.035 & 0.051 & 0.045 & 0.113 & 0.082 & $0.172 * *$ & $0.164 * *$ & $0.141 *$ \\
\hline
\end{tabular}

*P-value $<0.05$

$* *$ P-value $<0.01$

Citation: Talebi M, Zavar A, Ghafari M, et al. Improvement of quality of life and mental health in methadone maintenance therapy. MOJ Addict Med Ther. 2017;3(3):82-89. DOI: 10.15406/mojamt.2017.03.00039 


\section{Discussion and conclusion}

MMT improved significantly the quality of life of MMT outpatients $(1,9,16,19,27,28)$. In this study quality of life scores were shown to have improved in direct proportion to the MMT duration; nevertheless. Only the social subscale score was reported to be statistically significant, this was contrary to others findings, ${ }^{16,29,30}$ which can probably best explained by the fallacy on the part of addicts' family hoping for MMT to be a magic bullet. Other plausible reason why MMT may fail at times possibly regards unemployment as a result of social alienation, discrimination and stigma against drug users. In our attempt to identify the intervening as well as influential parameters on QoL scores, we managed to come up with employment, family and education statues as the top three of the list, studies which is also corroborated by other MMT has been shown to improve the QoL of those who have received on an outpatient basis. ${ }^{1,27}$

Our study declared mental health was improved by MMT. There was a decrease in all subscales of "Depression Anxiety Stress Scale". Mental health is allegedly poor among drug addicts, with low selfesteem and anxiety prevailing, compared to non-addicts. MMT enabled patients to feel less stressed as therapy commenced owing to the family support they benefit from, as well as the obviation of regarding arrest and hospitalization for overdose. Some studies demonstrated that MMT continually improved outpatients' mental health. 1,9,27,30 Also employment, marriage, health (affected by an underlying chronic disease) and education are the most important factors that may affect DASS subscales scores.

MMT improved the clients' ability to work. Finding a job is the vital first step for drug users to reconstitute their social integration. This suggests a need to provide occupation training programs in MMT clinics and conduct interventions in societies to reduce stigma against drug addicts.

This study has made available small but important evidence in favor MMT programme effectiveness. Yet it only regarded Qol assessment scores, leaving to additional qualitative evaluation such as in depth interview with the clients for farther investing action. We forestall that the positive trend in improvement in QoL will continue. More studies are needed to confirm that the improvement in the quality of life will sustain in the long run, thus longer cohort to studies are recommended to monitor the trend.

\section{Acknowledgements}

This study has been supported by Addiction Research Center of Mashhad University of Medical Sciences. The cooperation of all participants is highly appreciated.

\section{Conflict of interest}

The author declares no conflict of interest.

\section{References}

1. Baharom N, Hassan MR, Ali N, et al. Improvement of quality of life following 6 months of methadone maintenance therapy in Malaysia. Subst Abuse Treat Prev Policy. 2012;7:32.

2. National Institute Of Drug Abuse. Advancing addiction science. 2007.

3. Afshari R, Majdzadeh R, Balali-Mood M. Pattern of acute poisoning in Mashhad, Iran 1993-2000. J Toxicol Clin Toxicol. 2004;42(7):965-975.

4. Karami M, Etemadifard S. Social Structure of Addiction in Iran. Iranian Journal of Social Problems. 2011;1(4):93-110.
5. Zavar A, Afshari R, Alidoust M, et al. Curriculum Development in regard to Illicit Drug Abuse. FMEJ. 2012;2(2):31-36.

6. World Drug Report. United Nations Office on Drugs and Crime. Vienna, USA; 2010. p. 1-313.

7. World Drug Report. United Nations Office on Drugs and Crime. Vienna, USA; 2011. p. 1-272.

8. World Drug Report. United Nations Office on Drugs and Crime, Vienna, USA; 2008. p. 1-310.

9. Chou YC, Shih SF, Tsai WD, et al. Improvement of quality of life in methadone treatment patients in northern Taiwan: a follow-up study. BMC psychiatry. 2013;13:190 p.

10. Grella CE, Lovinger K. 30-year trajectories of heroin and other drug use among men and women sampled from methadone treatment in California. Drug and alcohol depend. 2011;118(2-3):251-258.

11. Literature Review-Methadone Maintenance Treatment. Office of Canada's Drug Strategy-Health Canada 2013. 2002.

12. World Health Organization. Proposal for the inclusion of Methadone in the WHO model list of essential medicines. 2004.

13. World Health Organization. The World Health Organization Quality of Life (WHOQOL). 2013.

14. Fitzpatrick R, Fletcher A, Gore S, et al. Quality of life measures in health care. I: Applications and issues in assessment. BMJ. 1992;305(6861):1074-1077.

15. Mazaheri M. The QOL-DASS Model to Estimate Overall Quality of Life and General Subjective Health. Iran J Psychiatry. 2011;6(1):43-46.

16. Vanagas G, Padaiga Z, Subata E. Drug addiction maintenance treatment and quality of life measurements. Medicina (Kaunas). 2004;40(9):833841.

17. Development of the World Health Organization WHOQOL-BREF quality of life assessment. The WHOQOL Group. Psychol Med. 1998;28(3):551-558.

18. World Health Organization. WHO Quality of Life-BREF (WHOQOL-BREF), USA; 2013.

19. Xiao L, Wu Z, Luo W, et al. Quality of life of outpatients in methadone maintenance treatment clinics. J Acquir Immune Defic Syndr. 2010;53(Suppl 1):116S-120S.

20. Nejat S, Montazeri A, Holakouie Naieni K, et al. The World Health Organization quality of Life (WHOQOL-BREF) questionnaire: Translation and validation study of the Iranian version. Journal of School of Public Health and Institute of Public Health Research. 2006;4(4):1-12.

21. Yousefy AR, Ghassemi GR, Sarrafzadegan N, et al. Psychometric properties of the WHOQOL-BREF in an Iranian adult sample. Community Ment Health J. 2010;46(2):139-147.

22. SH Lovibond, PF Lovibond. Manual for the depression anxiety stress scales. Psychology Foundation, Sydney, Australia; 1995. 42 p.

23. Ghassamia M, Asghari A, Shaeiri MR, et al. Validation of psychometric properties of the persian version of the female sexual function index. Urol J. 2013;10(2):878-885.

24. Mahmoudi Gharaei J, Mostafavi S, Alirezaei N. Quality of Life and the Associated Psychological Factors in Caregivers of Children with PKU. Iran J Psychiatry. 2011;6(2):66-69.

25. Yazdani M, Rezaei S, Pahlavanzadeh S. The effectiveness of stress management training program on depression, anxiety and stress of the nursing students. Iran J Nurs Midwifery Res. 2011;15(4):208-215.

26. Dodding C, Nasel D, Murphy M, et al. All in for mental health: a pilot study of group therapy for people experiencing anxiety and/or depression and a significant other of their choice. Ment Health Fam Med. 2008;5(1):41-49. 
27. Rohani S, Salarieh I, Abedi S, et al. The effect of MMT on the quality of life for people dependent on drugs. Journal of Mazandaran University of Medical Sciences. 2012;22(87):47-55.

28. Torrens M, Domingo Salvany A, Alonso J, et al. Methadone and quality of life. Lancet. 1999;353(9158):1101.

29. Padaiga Z, Subata E, Vanagas G. Outpatient methadone maintenance treatment program. Quality of life and health of opioid-dependent persons in Lithuania. Medicina (Kaunas). 2007;43(3):235-241.
30. Xiao L, Wu Z, Pang L. Qualitative study on the effectiveness of community-based methadone maintenance treatment among heroin addicts in some regions of China. Chinese Journal of AIDS \& STD. 2007;13:195197. 